University of Rhode Island

DigitalCommons@URI

Mechanical, Industrial \& Systems Engineering

Faculty Publications

2016

\title{
Dynamic Model for Fatigue Evolution in a Cracked Beam Subjected to Irregular Loading
}

\author{
Son Hai Nguyen \\ University of Rhode Island \\ David Chelidze \\ University of Rhode Island, chelidze@uri.edu
}

Follow this and additional works at: https://digitalcommons.uri.edu/mcise_facpubs

The University of Rhode Island Faculty have made this article openly available.

Please let us know how Open Access to this research benefits you.

This is a pre-publication author manuscript of the final, published article.

Terms of Use

This article is made available under the terms and conditions applicable towards Open Access

Policy Articles, as set forth in our Terms of Use.

\section{Citation/Publisher Attribution}

Hai Nguyen S, Chelidze D. Dynamic Model for Fatigue Evolution in a Cracked Beam Subjected to Irregular Loading. ASME. J. Vib. Acoust. 2016;139(1):014502-014502-6. doi:10.1115/1.4035112.

Available at: http://dx.doi.org/10.1115/1.4035112

This Article is brought to you for free and open access by the Mechanical, Industrial \& Systems Engineering at DigitalCommons@URI. It has been accepted for inclusion in Mechanical, Industrial \& Systems Engineering Faculty Publications by an authorized administrator of DigitalCommons@URI. For more information, please contact digitalcommons-group@uri.edu. 


\title{
Dynamic Model for Fatigue Evolution in a Cracked Beam Subjected to Irregular Loading
}

\author{
Son Hai Nguyen and David Chelidze* \\ Department of Mechanical, Industrial and Systems Engineering \\ University of Rhode Island, Kingston, RI 02881
}

The coupling of vibration and fatigue crack growth in a simply supported uniform Euler-Bernoulli beam containing a single-edge crack is analyzed. The fatigue crack length is treated as a generalized coordinate in a model for the mechanical system. This coupled model accounts for the interaction between the beam oscillations and the crack propagation dynamics. Nonlinear characteristics of the beam motion are introduced as loading parameters to the fatigue model to match experimentally observed failure dynamics. The method of averaging is utilized both as an analytical and numerical tool to: (1) show that, for cyclic loading, our fatigue model reduces to the Paris' law, and (2) compare the predicted fatigue damage accumulation with the experimental data for chaotic and random loadings. A utility of the fatigue model is demonstrated in estimating fatigue life under irregular loadings.

\section{Introduction}

The prediction of fatigue crack growth is a major problem of engineering practice. Various solutions to this problem have been proposed and are summarized in Refs. [1,2]. These approaches vary from continuum damage theories to linear damage rules. However, the industrial state of the art relies on the rain-flow counting method and the PalmgrenMiner rule [3-5], which ignore load interaction effects (e.g., crack growth retardation due to overloads [6]). On the other hand, studies of dynamic behavior of damaged structures [7] usually assume damage is a constant factor. In fact, the fatigue process is not only driven by environmental factors and structural dynamics, but it also affects structural dynamics by altering structural parameters. Recent damage models [8,9] describe the behavior of cracks at microscopic scale but they are not directly applicable in structural health monitoring and condition based maintenance technologies at macro-scale of structures. The aim of this paper is to couple the damage evolution and structural dynamics at macroscopic scale. Us-

*Address all correspondence to this author. Email: chelidze@uri.edu ing the proposed model, the fatigue crack propagation can be predicted from easily measurable structural vibration. Here, our system of interest is a cracked Euler-Bernoulli beam which is well studied in literature and our approach is based on a modification of the one-dimensional model of nonlinear damage dynamics described in Refs. [10,11].

Fatigue in materials is a nonlinear process in which the fatigue life is nonlinearly coupled with load factors $[12,13]$. Current fatigue damage models only take into account basic load statistical quantities such as mean, variance, maximum and minimum. These simple features do not capture important nonlinear characteristics of the applied loads [14, 15] (e.g., Lyapunov exponent, correlation dimension). In the next section, loading rate and divergence rate are introduced as loading parameters to the dynamic model of a cracked Euler-Bernoulli beam. Then the method of averaging is used to show our model reduces to Paris' law for cyclic loadings, and to determine the model's free parameters. Finally, the proposed fatigue model is validated by contrasting its predictions with the experimentally measured fatigue crack growth in aluminum beams excited by stochastic and chaotic signals possessing similar spectral and statistical characteristics.

\section{Equations of motion for a cracked beam}

A simply supported Euler-Bernoulli beam with a crack is shown in Fig. 1. Let $w(x, t)$ denote the deflection of the beam at spatial coordinate $x$ and time $t$. The normalized crack length $\phi(t)$ at $x=L / 2$ represents the current damage state,

$$
\phi=\frac{a}{b}
$$

where $a$ is the crack length, and $b$ is the width of the beam, $0 \leq \phi \leq 1$ ( 0 for the undamaged state and 1 for fractured). In this study we assume damage accumulation only reduces the beam's bending stiffness $[16,17]$. Then the potential and kinetic energies are determined by classical beam theory. The 


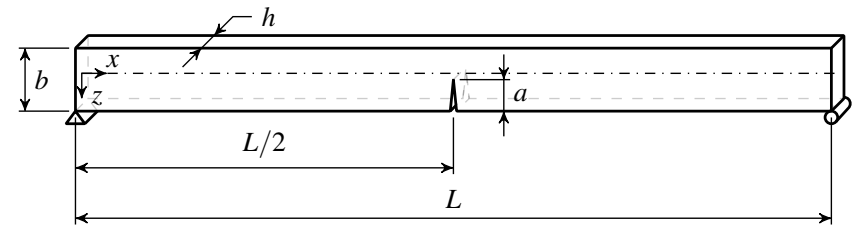

Fig. 1: Geometry of a simply supported beam with an edge crack

virtual work done by nonconservative forces can be written as

$$
\delta W=\int_{0}^{L}(F(x, t) \delta w-c \dot{w} \delta w-\Phi(\phi, \dot{\phi}) \delta \phi) \mathrm{d} x
$$

where $F(x, t)$ is the body force per unit length, $c \dot{w}$ is linear structural damping, and $\Phi(\phi, \dot{\phi}) \geq 0$ is the generalized force associated with the damage variable $\phi$, the overdot denotes differentiation with respect to time $t$. We expect damage growth will dissipate energy, therefore, the $\Phi$ term is kept negative. The coupling between structural vibration and fatigue crack growth is constructed through the application of Hamiltons principle as described in Refs. $[10,11]$

$$
\begin{aligned}
m_{\rho} \ddot{w}+\left(\bar{E} \bar{I} w^{\prime \prime}\right)^{\prime \prime}+c \dot{w} & =F(x, t), \\
\frac{1}{2} w^{\prime \prime 2} \frac{\partial \bar{E} \bar{I}}{\partial \phi}+\Phi & =0,
\end{aligned}
$$

where $m_{\rho}(x)$ is the mass per unit length of the beam, $\bar{E} \bar{I}=$ $\bar{E} \bar{I}(x, \phi)$ is the effective stiffness. The prime denote differentiations with respect to $x$. The partial differential equations will also have the corresponding boundary conditions $w(0, t)=w^{\prime \prime}(0, t)=0$ and $w(L, t)=w^{\prime \prime}(L, t)=0$.

Eq. (3a) describes the transverse vibration of the beam, in which the damage accumulation alters the stiffness of the beam. Eq. (3b) reflects the evolution of the damage $\phi$ under the beam's vibration and $\Phi$.

\section{The Effective Stiffness}

In this paper, we consider crack is always open. The presence of a crack changes the stiffness distribution in the vicinity of the cracked cross-section. For a rectangular section beam the effective stiffness has the following form [16]:

$$
\bar{E} \bar{I}(\phi, x)=E I\left\{1+\left[(1-\phi)^{-3}-1\right] \mathrm{e}^{\phi|x-L / 2|}\right\}^{-1},
$$

where $E$ and $I$ are the elastic modulus and the second moment of area of the undamaged beam, respectively. At the cracked cross-section, $x=L / 2$, the beam stiffness becomes

$$
\bar{E} \bar{I}(\phi, L / 2)=E I(1-\phi)^{3} .
$$

\section{Damage Growth Rate Law}

In Ref [18], we have showed that standard linear damage Palmgren-Miner law and Rainflow-counting method failed to estimate damage during fatigue testing under chaotic loading, while being adequate for random loading, which had the same statistical and spectral properties as chaotic. For deterministic loads, these experiments indicated that fatigue life depends not only on the number of stress cycles but also on the divergence rate $\lambda$, which measures the average rate of the expansion and folding process in an attractor. The higher $\lambda$, the faster the crack tip escapes the plastic zone due to an overload. $\lambda$ is estimated by the slope of the average trajectory divergence curve defined in Ref. [18].

The evolution model for $\phi$ can be obtained by making $\Phi$ explicitly depend on $\dot{\phi}$ in Eq. (3b). Since this model is formulated in terms of actual time not cycles, the fatigue life will depend on the rate or frequency of the applied loads. To characterize this dependence of $\Phi$ on the time rate of irregular loadings, $\sigma$, we introduce the loading rate parameter

$$
R_{\sigma}=\sqrt{\frac{\left\langle\dot{\sigma}^{2}\right\rangle}{\left\langle\sigma^{2}\right\rangle}}
$$

where $\langle x\rangle$ means the expectation value of $x$. For a cyclic stress $\sigma=\Delta \sigma \cos (\omega t) / 2, R_{\sigma}=\omega$.

To account for the nonlinear characteristics of the applied load and to obtain the correspondence between our model and Paris' law, $\Phi$ is assumed an explicit function of $R_{\sigma}$ :

$$
\Phi\left(\phi, \dot{\phi}, R_{\sigma}\right)=\frac{1}{\eta(\lambda)} \frac{f(\phi) \dot{\phi}^{p}}{R_{\sigma}^{q}}
$$

where $p, q$ are some positive exponents and $\lambda$ is the divergence rate of the applied load.

$$
\text { At } x=L / 2 \text {, substituting } w^{\prime \prime}(L / 2)=\frac{2 \sigma}{b E} \text { and Eq. (5) into }
$$

Eq. (3b) yields:

$$
\Phi\left(\phi, \dot{\phi}, R_{\sigma}\right)=\frac{6 I}{b^{2} E}(1-\phi)^{2} \sigma^{2} .
$$

Solving Eq. (7) for $\dot{\phi}$, we obtain a crack propagation model:

$$
\dot{\phi}=\left[\frac{6 \eta(\lambda) I}{b^{2} E}\right]^{\frac{1}{p}} R_{\sigma}^{\frac{q}{p}} f(\phi)^{-\frac{1}{p}}(1-\phi)^{\frac{2}{p}} \sigma^{\frac{2}{p}} .
$$


For the damage model derived above to be valid Eq. (9) should reduce to with Paris' law under cyclic load $\sigma=$ $\Delta \sigma \cos (\omega t) / 2$. In particular, substituting $R_{\sigma}=\omega$ and $\lambda=0$ into Eq. (9) we get

$$
\dot{\phi}=\left[\frac{6 \eta(0) I}{b^{2} E}\right]^{\frac{1}{p}} \omega^{\frac{q}{p}} f(\phi)^{-\frac{1}{p}}(1-\phi)^{\frac{2}{p}} \Delta \sigma^{\frac{2}{p}}\left(\cos (\omega t)^{2}\right)^{\frac{1}{p}}
$$

or

$$
\dot{\phi}=C_{0} \omega^{\frac{q}{p}} f(\phi)^{-\frac{1}{p}}(1-\phi)^{\frac{2}{p}} \Delta \sigma^{\frac{2}{p}}\left(\cos (\omega t)^{2}\right)^{\frac{1}{p}},
$$

where all material constants and systems parameters have been merged into the constant $C_{0}$. Since a fatigue crack accumulates slowly (e.g., a crack grows over thousands of load cycles), we can apply the method of averaging [19] to Eq. (11) yielding

$$
\dot{\phi} \approx C_{0} \omega^{\frac{q}{p}} f(\phi)^{-\frac{1}{p}}(1-\phi)^{\frac{2}{p}} \Delta \sigma^{\frac{2}{p}}\left(\frac{\omega}{2 \pi} \int_{0}^{\frac{2 \pi}{\omega}}\left(\cos (\omega t)^{2}\right)^{\frac{1}{p}} \mathrm{~d} t\right),
$$

or

$$
\dot{\phi} \approx C_{1} \omega^{\frac{q}{p}} f(\phi)^{-\frac{1}{p}}(1-\phi)^{\frac{2}{p}} \Delta \sigma^{\frac{2}{p}},
$$

where $C_{1}=C_{0} I_{d}$, and $I_{d}$ is the value of the integral inside the brackets of Eq. (12). Changing to a new time variable $N=\omega t /(2 \pi)$ and letting $\phi=a / b$, Eq. (13) can be rewritten to get

$$
\frac{\mathrm{d} a}{\mathrm{~d} N} \approx C_{2} \omega^{\frac{q}{p}-1} f(\phi)^{-\frac{1}{p}}(1-\phi)^{\frac{2}{p}} \Delta \sigma^{\frac{2}{p}}
$$

where $C_{2}=2 \pi b C_{1}$ is a constant.

On the other hand, Paris' law under cyclic load is given by

$$
\frac{\mathrm{d} a}{\mathrm{~d} N}=C\left(\Delta K_{I}\right)^{m}
$$

where $N$ is the number of load cycles, $C$ and $m$ are material constants. $\Delta K_{I}$ is the range of the stress intensity factor, and it can be expressed as $K_{I}=\sigma \sqrt{\pi a} Y(\phi) . Y(\phi)$ a function that depends on the geometry. Then Eq. (15) becomes

$$
\frac{\mathrm{d} a}{\mathrm{~d} N}=C_{3}[\sqrt{\phi} Y(\phi)]^{m} \Delta \sigma^{m}
$$

where $C_{3}=C(\pi b)^{\frac{m}{2}}$.

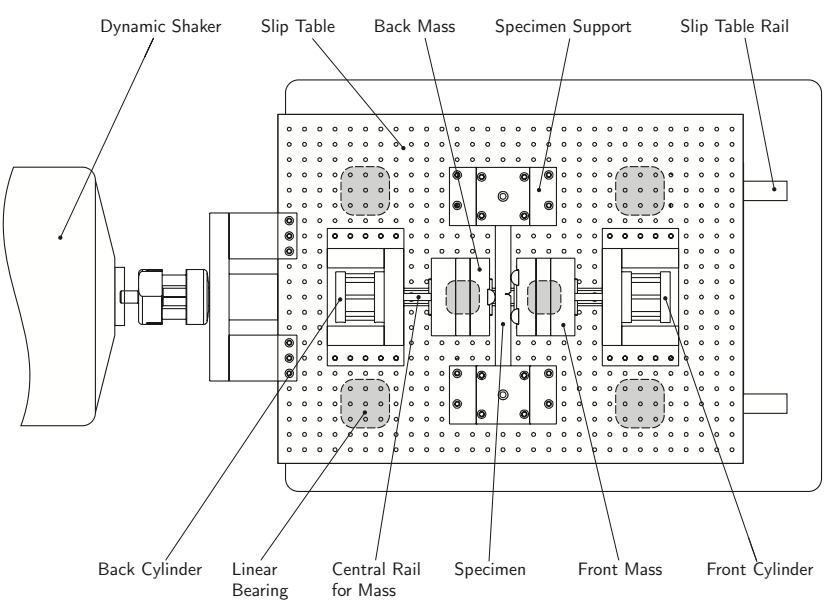

Fig. 2: Schematic of the apparatus

Comparing the exponents of $\omega, \Delta \sigma$, and the functions of $\phi$ in Eqs. (14) and (16) yields

$$
\begin{aligned}
\frac{q}{p} & =1 \rightarrow q=p, \\
\frac{2}{p} & =m \rightarrow p=\frac{2}{m}, \\
f(\phi) & =\left[\frac{\sqrt{\phi} Y(\phi)}{1-\phi}\right]^{-m p} .
\end{aligned}
$$

Thus, the derived damage model reduces to Paris' law for cyclic loads provided the parameters satisfy Eqs. (17)-(19). In the following experiment, the specimen is made from aluminum which has the material constant $m \approx 3$ [20]. By setting $m=3$, we get $q=p=\frac{2}{3}$, and $f(\phi)=\left[\frac{\sqrt{\phi} Y(\phi)}{(1-\phi)}\right]^{-2}$.

\section{Experimental validation}

The fatigue experiments described in Ref. [18] are used to validate the new fatigue model under irregular, non-cyclic loadings. The details of the experimental system are given in Refs. [18,21], and a schematic of the test rig is shown in Fig. 2. The masses are kept in contact with the specimen by two pneumatic cylinders. The main operating principle of the rig is that inertial forces of masses generated by the electromagnetic shaker provide dynamic loads to the specimen. The specimen is a single edge notched beam which is simply supported by pins on each end.

The specimens are designed to follow the fracture toughness test standard ASTM E1820-08a [22] and their model is shown in Fig. 3. The specimen is made of 6061 aluminum bar stock, with dimensions $314.70 \times 20.32 \times 6.35 \mathrm{~mm}$, and the fatigue is initiated by a machined ' $\mathrm{V}$ ' notch. In order to 


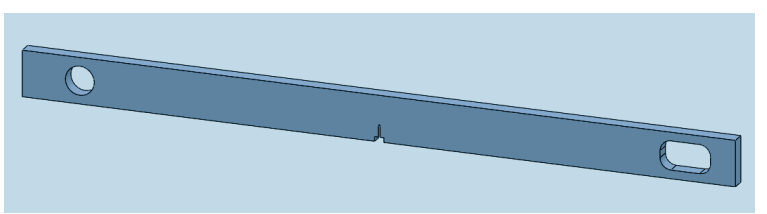

Fig. 3: Model of the specimen. The machined notch is at the center.
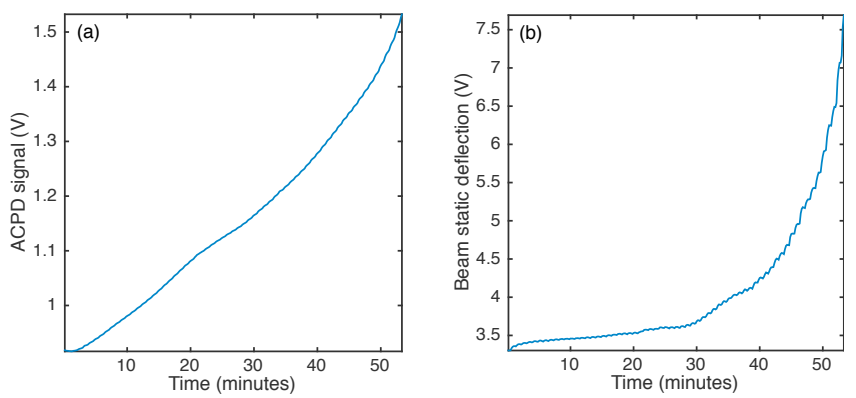

Fig. 4: Measurements of fatigue crack (a) and beam static deflection (b).

keep the beam vibration small and the duration of the experiment not too long, the notch is cut to a depth of $7.62 \mathrm{~mm}$ by a blade $0.762 \mathrm{~mm}$ thick with a $60^{\circ} \mathrm{V}$-shaped tip. The initial crack length was examined under a Stocker and Yale optical micrometer at $40 \times$ magnification. The crack lengths of all the fatigue tests recorded were measured to be $7.823 \mathrm{~mm} \pm$ $0.069 \mathrm{~mm}$ which yielded the initial damage $\phi_{0}=0.375$.

A chaotic signal and its random surrogate [23] are supplied to the shaker providing deterministic and stochastic excitations, respectively. The relative displacement between the slip table and a beam at $x=40 \mathrm{~mm}$ is measured by an eddy current sensor. The crack growth is monitored using an Alternating Current Potential Difference (ACPD) crack growth monitor. Fig. 4 shows the damage evolution (left) and the beam static deflection (right). It is clear that the propagating crack gradually reduces the beam stiffness which results in increased beam deflection.

The eddy current sensor measurements are used as inputs into the damage model and its predictions are compared with the crack length measurements coming from the ACPD monitor. The beam diagram and applied forces are shown in Fig. 5. The applied loads can be expressed as

$$
\begin{aligned}
& P_{1}=P-m a_{\mathrm{rel}}, \\
& P_{2}=\frac{P+m a_{\mathrm{rel}}}{2},
\end{aligned}
$$

where $P$ is the static mean force due to the pressure within the cylinders, and $a_{\mathrm{rel}}$ is the relative acceleration between the

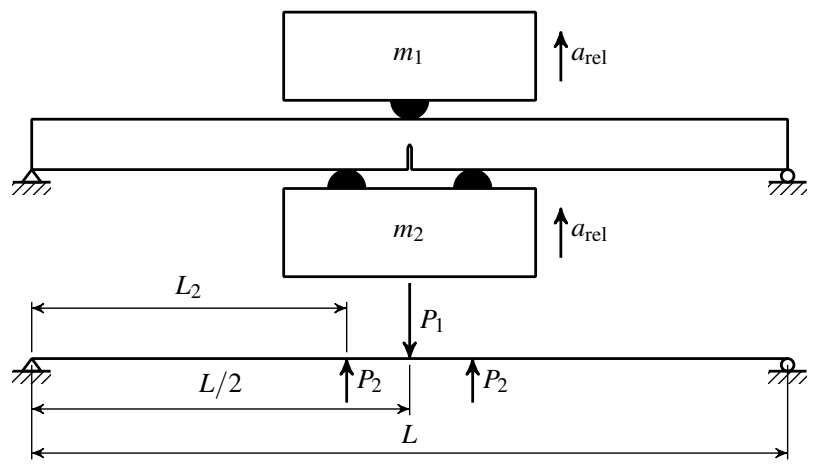

Fig. 5: Beam diagram with applied forces

masses and the slip table.

The force $F(x, t)$ in Eq. (3a) becomes

$F(x, t)=P_{1} \delta_{D}(x-L / 2)-P_{2} \delta_{D}\left(x-L_{2}\right)-P_{2} \delta_{D}\left(x-L+L_{2}\right)$,

where $\delta_{D}(x)$ is the Dirac delta function.

In our setup, two masses are always kept contact with the beam and they only move back and forth. Therefore, we consider a single mode motion

$$
w(x, t)=q(t) \psi(x)
$$

where $\psi(x)$ is the mode shape of the beam oscillations [24].

The range of the stress intensity factor $\Delta K_{I}$ is obtained from the principle of superposition [25]:

$$
\Delta K=m \frac{3 \sqrt{\pi} L}{\sqrt{b} h^{2}} \Delta a\left(\frac{1}{2} \sqrt{\phi} Y_{I}^{(1)}+\frac{L_{2}}{L} \sqrt{\phi} Y_{I}^{(2)}\right)
$$

where $Y_{I}^{(1)}$ and $Y_{I}^{(2)}$ are dimensionless functions [26]

$$
Y_{I}^{(1)}(\phi)=1.106-1.552 \phi+7.71 \phi^{2}-13.53 \phi^{3}+14.23 \phi^{4} ;
$$

and

$$
Y_{I}^{(2)}(\phi)=1.122-1.40 \phi+7.33 \phi^{2}-13.08 \phi^{3}+14.0 \phi^{4} .
$$

Then, the function $f(\phi)$ in Eq. (9) has the following form:

$$
f(\phi)=\left(\frac{1}{2} \frac{\sqrt{\phi}}{(1-\phi)} Y_{I}^{(1)}+\frac{L_{2}}{L} \frac{\sqrt{\phi}}{(1-\phi)} Y_{I}^{(2)}\right)^{-2} .
$$


Table 1: Estimated divergence rates $\lambda$ and function $A(\lambda)$

\begin{tabular}{|c||c|c||c|c||}
\hline \hline \multirow{2}{*}{$\#$} & \multicolumn{2}{c||}{ Chaotic Loading } & \multicolumn{2}{c||}{ Random Loading } \\
\cline { 2 - 5 } & Div. rate $\lambda$ & $A(\lambda)$ & Div. rate $\lambda$ & $A(\lambda)$ \\
\hline 1 & $15.3 \pm 2.4$ & $29 \times 10^{-7}$ & $32.8 \pm 1.4$ & $50 \times 10^{-7}$ \\
\hline 2 & $15.0 \pm 2.6$ & $30 \times 10^{-7}$ & $32.4 \pm 1.4$ & $44 \times 10^{-7}$ \\
\hline 3 & $15.3 \pm 3.3$ & $32.5 \times 10^{-7}$ & $32.3 \pm 1.3$ & $45 \times 10^{-7}$ \\
\hline 4 & $15.3 \pm 2.7$ & $26.5 \times 10^{-7}$ & $32.3 \pm 1.2$ & $42 \times 10^{-7}$ \\
\hline 5 & $15.3 \pm 3.6$ & $32 \times 10^{-7}$ & $33.0 \pm 1.0$ & $47 \times 10^{-7}$ \\
\hline \hline
\end{tabular}

Using the relationship $w^{\prime \prime}(L / 2)=\frac{2 \sigma}{b E}$ and Eq. (22), the loading rate $R_{\sigma}$ can be determined by the recorded measurements of $w\left(L_{0}, t\right)$ (at $\left.L_{0}=40 \mathrm{~mm}\right)$ :

$$
R_{\sigma}=\sqrt{\frac{\left\langle\dot{\sigma}^{2}\right\rangle}{\left\langle\sigma^{2}\right\rangle}}=\sqrt{\frac{\left\langle\dot{w}_{x=L_{0}}^{2}\right\rangle}{\left\langle w_{x=L_{0}}^{2}\right\rangle}} .
$$

Finally, the damage growth rate in Eq. (9) can be written as

$$
\dot{\phi}=A(\lambda)\left(\frac{1}{2} \sqrt{\phi} F_{I}^{(b)}+\frac{L_{2}}{L} \sqrt{\phi} F_{I}^{(c)}\right)^{3} R_{\sigma}\left\langle w_{x=L_{0}}^{3}\right\rangle
$$

where $A(\lambda)=\left[\frac{6 E I \psi^{\prime \prime}(L / 2)^{2}}{\psi\left(L_{0}\right)^{2}} \eta(\lambda)\right]^{3 / 2}$ depends on material properties, geometry of the beam, the mode shape $\psi$, and the divergence rate $\lambda$.

In the following, the measurements of the displacement of the beam are used to advance and validate our model. In each test, the time series $w\left(L_{0}, t\right)$ are recorded at a $1 \mathrm{kHz}$ sampling rate, and are split into small data records, where each record contains 10,000 points. The estimated divergence rates are shown in Table 1. It is assumed that the changes in the damage variable over one data record (i.e., over a $10 \mathrm{~s}$ time span) are negligible due to its slow-time nature. The method of averaging is applied to simulate Eq. (28) by integrating over one data record. Thus, the fast time function $w\left(L_{0}, t\right)^{3}$ is replaced by its moving average $\left\langle w\left(L_{0}, t\right)^{3}\right\rangle_{t}$.

In Eq. (28), the function $A(\lambda)$ is unknown. However, the experiments are programmed to stop when the maximum stress is higher than the material yield strength $\left(\phi_{f} \approx 0.6\right)$. Using this condition and the initial damage $\phi_{0}=0.375$, the value of $A$ is adjusted so that the simulated final damage $\approx \phi_{f}$ as shown in Table 1. Thus, the focus here is on overall evolution trajectory and not on the initial and final trajectory points that were matched. The resulting damage trajectories from numerical simulations of Eq. (28) and the corresponding ACPD signals are normalized and shown in Fig. 6.

The experiment in Ref. [18] showed that the larger $\lambda$ of the applied load results in the higher crack growth rate. Especially when the system grows unbounded, $\lambda$ is expected to be a large number, and the failure of the system should happen immediately. Similar to the divergence rates $\lambda$, the matched values of $A(\lambda)$, as shown in Table 1 , are comparable for each individual loading type but distinct for different loading types. Therefore, $A$ is assumed to be an exponential function of $\lambda$ :

$$
A(\lambda)=\alpha e^{\beta \lambda}
$$

where $\alpha$ and $\beta$ are constants. To determine the values of $\alpha$ and $\beta$, three fatigue tests under a periodic load $(\lambda=0)$ were conducted and $A(0)$ was estimated. Then applying the exponential curve fitting yields $\alpha=20 \times 10^{-7}$ and $\beta=0.02$ (see Fig. 7).

\section{Predicting Time to Failure}

The ultimate goal of all fatigue crack propagation models is to predict the fatigue life of a system. If the applied load is known, our model can simulate the fatigue crack evolution in time and predict failure. However, it is not practical to monitor the response of a system for the whole fatigue life. The failure of a system should be estimated from the estimated future loading profile, which is usually assumed to remain similar to the one in the beginning of the fatigue life.

Here, our model described by Eq. (28) is used to estimate the time to failure (TTF) of the specimen assuming that the loading factors will remain the same as initially observed. In particular, the future loading rate $R_{\sigma}$, divergence rate $\lambda$, and $\left\langle w_{x=L_{0}}^{3}\right\rangle$ are determined from the first 200,000 points ( $\approx 3$ minutes of the fatigue life) of the displacement time series of the specimen at location $x=40 \mathrm{~mm}$. Then the TTF is calculated by integrating Eq. (28) from $\phi=0.375$ to $\phi=0.6$. Table 2 shows the estimated TTF for ten fatigue tests used in the previous section.

\section{Discussion}

The small differences between the real crack and simulated crack evolutions in Fig. 6 can be explained by the inherent variations/indeterminicity in the initial condition. In all tests reported here, we assume the initial damage to be the same, $\phi_{0}=0.375$. However, the actual initial cracks in each test are different due to notch fabrication tolerances and material irregularities effecting the microstructure of the beam. It is difficult, if not impossible, to accurately determine the size of the initial crack. In addition, in Eq. (28), environmental factors (e.g., temperature, humidity) are neglected in the analysis. 

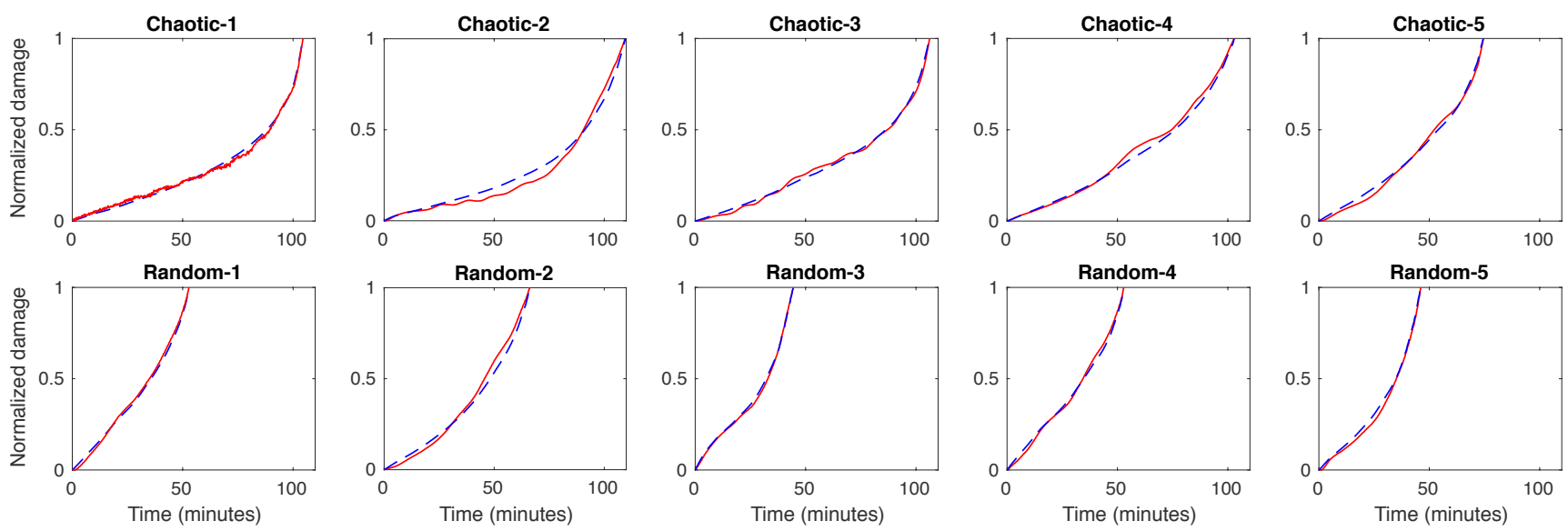

Fig. 6: Comparison of the ACPD signal (red -) with numerical integration (blue --)

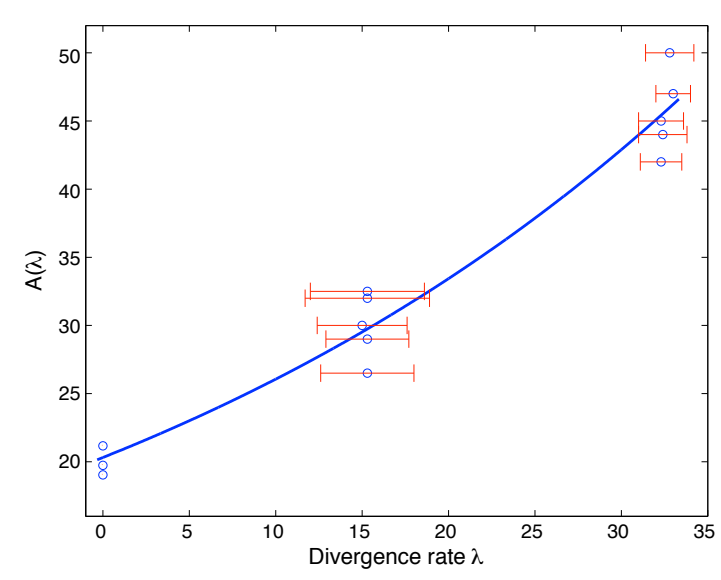

Fig. 7: Exponential curve fitting.
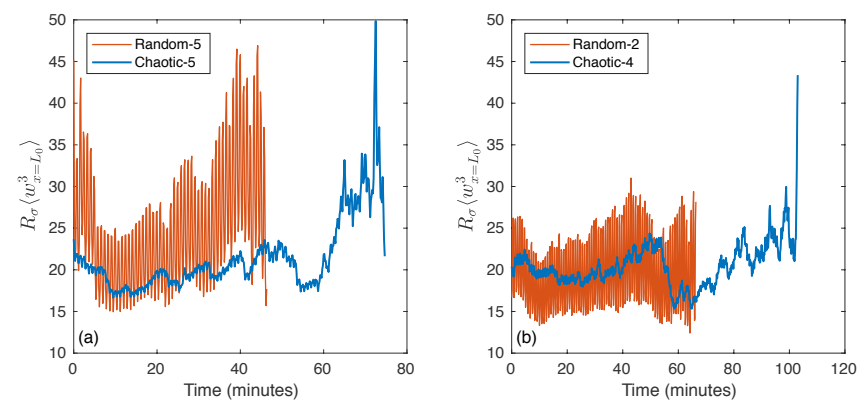

Fig. 8: Dependence of $R_{\sigma}\left\langle w_{x=L_{0}}^{3}\right\rangle$ in time.

For the fatigue life prediction, the difference between the TTF and the estimated TTF is mainly caused by the changes in the estimated load parameters. The growing crack changes not only the system parameters but also the applied load to the specimen. Fig. 8 shows how the val-
Table 2: True and estimated Times to Failure

\begin{tabular}{||c|c|c||c|c|c||}
\hline \hline \multicolumn{3}{|c||}{ Chaotic Loading } & \multicolumn{3}{c||}{ Random Loading } \\
\hline $\begin{array}{c}\text { True TTF } \\
\text { (hrs:min) }\end{array}$ & $\begin{array}{c}\text { Est. TTF } \\
\text { (hrs:min) }\end{array}$ & Error & $\begin{array}{c}\text { True TTF } \\
\text { (hrs:min) }\end{array}$ & $\begin{array}{c}\text { Est. TTF } \\
\text { (hrs:min) }\end{array}$ & Error \\
\hline $1: 44$ & $1: 53$ & $8 \%$ & $0: 53$ & $1: 10$ & $32 \%$ \\
$1: 50$ & $2: 21$ & $28 \%$ & $1: 07$ & $1: 06$ & $0 \%$ \\
$1: 46$ & $2: 01$ & $14 \%$ & $0: 44$ & $0: 55$ & $25 \%$ \\
$1: 42$ & $1: 43$ & $0 \%$ & $0: 52$ & $1: 00$ & $15 \%$ \\
$1: 13$ & $1: 41$ & $35 \%$ & $0: 45$ & $0: 54$ & $20 \%$ \\
\hline \hline
\end{tabular}

ues of $R_{\sigma}\left\langle w_{x=L_{0}}^{3}\right\rangle$ change in time. Fig. 8(a) corresponds to Random-5 and Chaotic-5 tests which have high estimated TTF errors as well as high variability in the loading factors. While Fig. 8(b) corresponds to Random-2 and Chaotic4 which have zero estimated TTF errors as well as low variability in loading factors.

In this paper, Paris' law is used as a special case to validate the fatigue model. Therefore, this model is only valid in Paris regime which requires long cracks and ignores the threshold limit, load ratio effects as well as effect of specimen thickness.

\section{Conclusion}

A new dynamic model of fatigue evolution was presented. Using the loading rate and divergence rate as loading parameters, the model can describe fatigue damage evolution under arbitrary loading, and also captures the interaction between the fatigue damage and structural dynamics. For cyclic loadings, our model reduced to Paris' law through 
the averaging method. The crack evolution was numerically simulated using the experimental measurements of structural vibration as input. The damage model predictions closely followed the actual, experimentally measured, crack growth trajectories in both chaotically and randomly forced tests. Then, the TTFs were estimated from the initial short-time response of the system and assuming the loading characteristics stayed the same as tests progressed. The initial results have shown that the proposed model can be used for estimating fatigue life, and the models simulation can accurately track actual crack growth if the nonlinear loading factors can be estimated and provided as input.

\section{Acknowledgments}

This paper is based upon work supported by the National Science Foundation under Grants No. 0758536 and 1100031 .

\section{References}

[1] A. Fatemi, L. Yang, Cumulative fatigue damage and life prediction theories: A survey of the state of the art for homogeneous materials, International Journal of Fatigue 20 (1) (1998) 9-34.

[2] A. Carpinteri (Ed.), Handbook of Fatigue Crack Propagation in Metallic Structures, Elsevier, 1994.

[3] M. Miner, Cumulative damage in fatigue, Journal of Applied Mechanics 67 (1945) A159-A164.

[4] A. Palmgren, Die lebensdauer von kugellagern, Verfahrenstechinik, Berlin 68 (1945) 339-341.

[5] E. Macha, T. Lagoda, A. Nieslony, D. Kardas, Fatigue life under variable-amplitude loading according to the cycle-counting and spectral methods., Materials Science 42(3) (2006) 416-425.

[6] H. L. Ewalds, R. J. H. Wanhill, Fracture Mechanics, Edward Arnold, 1984.

[7] A. D. Dimarogonas, Vibration of cracked structures: A state of the art review, Engineering Fracture Mechanics 55 (5) (1996) 831-857.

[8] C. Giry, F. Dufour, J. Mazars, Stress-based nonlocal damage model, International Journal of Solids and Structures 48 (2526) (2011) 3431-3443.

[9] G. Z. Voyiadjis, N. Mozaffari, Nonlocal damage model using the phase field method: Theory and applications, International Journal of Solids and Structures 50 (2021) (2013) 3136-3151.

[10] J. Cusumano, Q. Li, Coupled field damage dynamics via Hamilton's principle, ASME Conference Proceedings 2010 (44137) (2010) 771-776.

[11] J. Cusumano, A. Roy, Q. Li, Damage dynamics, rate laws, and failure statistics via Hamilton's principle, Meccanica 50 (1) (2015) 77-98.

[12] M. Skorupa, Load interaction effects during fatigue crack growth under variable amplitude loading - a literature review. Part I: Empirical Trends, Fatigue and Fracture of Engineering Materials and Structures 21 (1999) 987-1006.

[13] M. Skorupa, Load interaction effects during fatigue crack growth under variable amplitude loading - a literature review. Part II: Qualitative interpretations, Fatigue and Fracture of Engineering Materials and Structures 22 (1999) 905-926.

[14] H. Kantz, T. Schreiber, Nonlinear Time Series Analysis, Cambridge University Press, 2004.

[15] H. Abarbanel, Analysis of observed chaotic data, Institute for nonlinear science, Springer, 1996.

[16] S. Christides, A. Barr, One-dimensional theory of cracked Bernoulli-Euler beams, International Journal of Mechanical Sciences 26 (1984) 639-648.

[17] M. Shen, C. Pierre, Natural modes of bernoulli-euler beams with symmetric cracks, Journal of Sound and Vibration 138 (1990) 115-134.

[18] S. H. Nguyen, M. Falco, M. Liu, D. Chelidze, Different fatigue dynamics under statistically and spectrally similar deterministic and stochastic excitations, Journal of Applied Mechanics 81(4) (2014) 041004.

[19] J. A. Sanders, F. Verhulst, J. Murdock, Averaging Methods in Nonlinear Dynamical Systems, Springer, 2007.

[20] S. Suresh, Fatigue of Materials, Cambridge University Press, 1998.

[21] M. Falco, M. Liu, D. Chelidze, A new fatigue testing apparatus model and parameter identification, ASME 2010 International Design Engineering Technical Conferences and Computers and Information in Engineering Conference 5 (44137) (2010) 1007-1012.

[22] ASTM-E1820-08a, Standard Test Methods for Measurement of Fracture Toughness. Annual Book of ASTM Standards, American Society for Testing and Materials, Philadelphia, PA., 2008.

[23] T. Schreiber, A. Schmitz, Improved surrogate data for nonlinearity tests, Physical Review Letters 77 (1996) 635-638.

[24] L. Meirovitch, Principles and Techniques of Vibrations, Prentice Hall, 1996.

[25] T. L. Anderson, Fracture Mechanics: Fundamentals and Applications, CRC Press, Boca Raton, 1995.

[26] H. Tada, P. C. Paris, G. R. Irwin, The Stress Analysis of Cracks Handbook, American Society of Mechanical Engineers Press; Third edition, 2000. 